\title{
EVALUACIÓN DE FORMULACIONES DE KETOCONAZOL PARA USO TÓPICO EN LEISHMANIASIS CUTÁNEA POR Leishmania (Viannia)
}

\author{
Angélica M. Vera ${ }^{1, a}$, Omar A. Casadiego ${ }^{1, b}$, Julio C. Mantilla ${ }^{2, c}$, Patricia Escobar $^{1, d}$
}

\begin{abstract}
RESUMEN
Se evaluó la efectividad de diversas formulaciones farmacéuticas de ketoconazol en modelos experimentales de leishmaniasis cutánea (LC) en ratones BALB C. Fueron preparadas formulaciones tópicas tipo gel, lipogel y crema conteniendo potenciadores de la permeación y diferentes concentraciones de ketoconazol. Se determinó la estabilidad, la toxicidad y la actividad anti-Leishmania in vitro. Además, se evaluó in vivo la efectividad de las formulaciones aplicadas tópicamente en ratones con LC infectados con Leishmania (Viannia) braziliensis. Las formulaciones tipo crema fueron evaluadas adicionalmente en ratones infectados con $L$. (V.) panamensis. Los sistemas evaluados mantuvieron in vitro la actividad del ketoconazol contra los parásitos; sin embargo, ninguna de las formulaciones fue efectiva en curar las lesiones de LC en los ratones. El tratamiento tópico con miltefosina (utilizado como control) curó las lesiones. Se concluye que las formulaciones que contienen ketoconazol diseñados en este estudio, no fueron efectivos contra la LC en los ratones infectados.
\end{abstract}

Palabras clave: Leishmania (Viannia) braziliensis; Leishmania (Viannia) panamensis; Ratones consanguíneos BALB C; Administración tópica (fuente: DeCS BIREME).

\section{EVALUATION OF KETOCONAZOLE FORMULATIONS FOR TOPICAL USE IN CUTANEOUS LEISHMANIASIS CAUSED BY Leishmania (Viannia)}

\begin{abstract}
The effectiveness of various pharmaceutical formulations of ketoconazole was evaluated in experimental models of cutaneous leishmaniasis (LC) in BALB C mice. Topical gel, lipogel, and cream formulations containing permeation enhancers and different concentrations of ketoconazole were prepared. Stability, toxicity and anti-Leishmania activity were determined in vitro. In addition, the effectiveness of topically applied formulations in LC-infected mice infected with Leishmania (Viannia) braziliensis was evaluated in vivo. Cream formulations were additionally evaluated in mice infected with $L$. (V.) panamensis. The systems evaluated maintained in vitro the activity of ketoconazole against parasites; however, none of the formulations were effective in curing LC lesions in mice. Topical treatment with miltefosine (used as a control) cured the lesions. It is concluded that the ketoconazole-containing formulations designed in this study were not effective against LC in infected mice.
\end{abstract}

Keywords: Leishmania (Viannia) braziliensis; Leishmania (Viannia) panamensis; Inbred BALB C mice; Topical administration (source: MeSH NLM).

\section{INTRODUCCIÓN}

La leishmaniasis son un grupo de enfermedades causadas por parásitos intracelulares del género Leishmania, transmitida a los humanos por insectos flebótomos. Sus manifestaciones clínicas varían según la especie de parásito involucrada y el estado inmune del hospedero, desde leishmaniasis visceral hasta formas asintomáticas o leves de leishmaniasis cutánea (LC) ${ }^{(1)}$. La leishmaniasis del Nuevo Mundo (especialmente producida por especies del subgénero Viannia) constituye la forma más polimórfica de la enfermedad, encontrándose distribuida ampliamente en Brasil, Colombiay Perú (2). El tratamiento está basado en el uso parenteral de antimoniales pentavalentes, y en caso de falla

\footnotetext{
1 Centro de Investigaciones en Enfermedades Tropicales (CINTROP), Escuela de Medicina, Departamento de Ciencias Básicas, Universidad Industrial de Santander. Bucaramanga, Colombia

2 Grupo PAT-UIS. Escuela de Medicina, Departamento de Patología, Universidad Industrial de Santander. Bucaramanga, Colombia

a Microbióloga Industrial; ${ }^{\mathrm{b}}$ microbiólogo y bioanalista; ${ }^{\mathrm{c}}$ médico especialista en Patología; ${ }^{\mathrm{d}} \mathrm{PhD}$ en Inmunoparasitología

Recibido: 07/03/2018 Aprobado: 13/06/2018 En línea: 04/09/2018
}

Citar como: Vera AM, Casadiego OA, Mantilla JC, Escobar P. Evaluación de formulaciones de ketoconazol para uso tópico en leishmaniosis cutánea por Leishmania (Viannia). Rev Peru Med Exp Salud Publica. 2018;35(3):476-82. doi:10.17843/rpmesp.2018.353.3531. 
terapéutica o contraindicaciones se puede usar pentamidina, anfotericina B, sus formas liposomales o miltefosina. Algunos pacientes presentan efectos adversos serios como cardiopatías, nefropatías y pancreatitis. La eficacia del tratamiento se ve afectada por la especie de Leishmania, la edad del paciente, el estado inmune y el tiempo de evolución de las lesiones ${ }^{(3)}$. En pacientes con contraindicaciones los tratamientos tópicos son una alternativa atractiva, debido a que son de fácil administración, bajo costo y baja toxicidad; sin embargo, por el riesgo de diseminación, deben aplicarse con precaución y en combinación con otros fármacos ${ }^{(3)}$.

El ketoconazol (KTZ) es un imidazol de amplio espectro utilizado en el tratamiento de infecciones micóticas. Su uso oral ha sido restringido por la Food and Drug Administration (FDA) desde el $2013^{(4)}$; sin embargo, se encuentra disponible en diversos sistemas farmacéuticos (SF) de aplicación tópica como geles, cremas, aerosoles, espumas y champús (https://www.drugbank.ca/drugs/ DB01026) utilizados principalmente en infecciones fúngicas superficiales y en dermatitis seborreica. El KTZ es un inhibidor de la 14- $\alpha$ demetilasa (una enzima del citocromo P-450) necesaria en la conversión del lanosterol en ergosterol en hongos y Leishmania ${ }^{(5)}$

Algunos ensayos clínicos que han utilizado KTZ a $600 \mathrm{mg} /$ día/oral durante 28 días, han mostrado eficacias del $76 \%$ y $89 \%$ en el tratamiento de LC por L. (L.) mexicana y L. (V.) panamensis, respectivamente y del $30 \%$ en casos de LC por L. (V.) braziliensis ${ }^{(6-7)}$. Los resultados sobre su efectividad tópica (crema al $2 \%$ ) son pocos y desalentadores ${ }^{(8-10)}$. Nuevos sistemas farmacéuticos de ketoconazol (SFKTZ) en forma de espumas y geles han dado lugar a un «renacimiento» del uso de KTZ tópico para el tratamiento de enfermedades de la piel como dermatitis seborreica u otras entidades dermatológicas ${ }^{(11)}$. Igualmente, los SF-KTZ conteniendo potenciadores de la permeación percutánea (PPP) (p. ej. surfactantes, terpenos) y nuevos sistemas de administración de fármacos como liposomas, niosomas y microemulsiones están siendo evaluados tratando de aumentar su eficacia y reducir sus efectos secundarios ${ }^{(12)}$.

El objetivo de este trabajo fue diseñar y evaluar la efectividad de diversos SF-KTZ conteniendo diversos PPP en modelos experimentales de LC en ratones BALB C.

\section{EL ESTUDIO}

\section{PREPARACIÓN Y CARACTERIZACIÓN}

Se prepararon SF-KTZ tipo gel, cremas y lipogeles que contienen liposomas ultradeformables (LUD) con o sin KTZ o PPP (Tabla 1). Para los geles, el Carbopol $940 ®(1 \%$ p/p) se disolvió en agua destilada y posteriormente se agregó el benzoato sódico al 0,1\%, el KTZ y los PPP por agitación constante. La solución fue neutralizada con TEA hasta formar un gel transparente. Para las cremas, la fase acuosa (agua, glicerina, trietanolamina y benzoato de sodio) fue mezclada con la fase oleosa (ácido esteárico y cutina) en

\section{MENSAJES CLAVE}

Motivación para realizar el estudio. Las formulaciones de aplicación tópica en piel constituyen una alternativa de tratamiento para la leishmaniasis cutánea (LC) en pacientes que presentan contraindicaciones al tratamiento habitual con antimoniales.

Principales hallazgos. Los sistemas farmacéuticos conteniendo ketoconazol (SF-KTZ) evaluados in vitro fueron efectivos en el tratamiento de LC; sin embargo, ninguno de los SF-KTZ aplicados tópicamente logro curar a los ratones infectados con LC.

Implicancias. Es necesario la investigación de nuevos sistemas farmacéuticos que permitan una mejor absorción del ketoconazol en modelos experimentales con ratones.

agitación continua a $75^{\circ} \mathrm{C}$ en baño María. Posteriormente se agregó el KTZ, los PPP y la vitamina E hasta obtener una homogenización completa. Los LUD con KTZ fueron preparados utilizando el método de hidratación de una película fina según lo descrito por Hernández et al. ${ }^{(13)}$. Los liposomas fueron adicionados a los geles hasta obtener un sistema homogéneo.

Los SF-KTZ y sus vehículos fueron caracterizados determinándose su color, aspecto y $\mathrm{pH}$ a $4^{\circ} \mathrm{C}, 28^{\circ} \mathrm{C}$ y $37^{\circ} \mathrm{C}$ por 30 días. El tamaño de partícula, potencial zeta y el índice de polidispersión (PDI) de los LUD fue determinado utilizando un Zetasizer (Malvern Nano ZS® 90).

\section{PARÁSITOS Y CÉLULAS}

Los promastigotes de L. (V.) braziliensis (MHOM/BR/75/ M2903) y L. (V.) panamensis (MHOM/PA/71/LS94) fueron cultivados en medio Schneider suplementado con $10 \%$ de suero fetal bovino e inactivado con calor (SFBi) a $28^{\circ} \mathrm{C}$. La línea celular monocítica humana (THP-1, ATCC TIB-202) fue mantenida en medio de cultivo RPMI 1640 y $10 \%$ de SBFi a $37{ }^{\circ} \mathrm{C}, 5 \%$ de $\mathrm{CO}_{2}$ y $95 \%$ de humedad. Los amastigotes intracelulares se obtuvieron infectando las células THP-1 con promastigotes en una relación célula: parásito de 1:5 por 48 horas ${ }^{(13)}$.

Tabla 1. Sistemas farmacéuticos utilizados en el estudio

\begin{tabular}{|c|c|c|c|c|c|}
\hline \multirow{2}{*}{$\begin{array}{l}\text { Sistema } \\
\text { farmacéutico }\end{array}$} & \multirow{2}{*}{$\begin{array}{l}\text { KTZ } \\
(\%)\end{array}$} & \multicolumn{3}{|c|}{$\begin{array}{c}\text { Potenciadores de la } \\
\text { permeación (\%) }\end{array}$} & \multirow{2}{*}{$\begin{array}{c}\text { Vitamina } \\
E^{*} \\
(\%)\end{array}$} \\
\hline & & $\begin{array}{l}\text { Ácido } \\
\text { oleico }\end{array}$ & $\begin{array}{c}\text { trans- } \beta- \\
\text { cariofileno }\end{array}$ & DMSO & \\
\hline Gel 1 & 1 & - & 0,5 & 10 & - \\
\hline Gel 2 & 1 & 0,8 & - & 10 & - \\
\hline Gel 3 & 1,5 & - & - & 20 & - \\
\hline Gel 4 & 1,5 & - & - & & - \\
\hline Lipogel & 1 & 1 & - & 6 & - \\
\hline Cremas (1-4) & $n$ & 1,7 & 0,5 & - & 1 \\
\hline
\end{tabular}

KTZ: ketoconazol, DMSO: dimetilsulfoxido, n: cremas con diferentes concentraciones de ketoconazol (2\% a $15 \%$ )

* A los sistemas farmacéuticos tipo crema se agregó $1 \%$ de vitamina $E$ como antioxidante 


\section{ENSAYOS IN VITRO}

Los parásitos y células fueron tratados con diluciones seriadas 1:3 de KTZ libre (en solución) y los SF-KTZ durante 72 horas. Las células control fueron mantenidas sin compuesto. La inhibición del crecimiento parasitario fue determinada microscópicamente por conteo directo de promastigotes o por recuento de células infectadas con amastigotes. La toxicidad en las células THP-1 fue determinada utilizando el método colorimétrico de bromuro de 3-(4,5-dimetiltiazol-2-il)-2,5-difeniltetrazolio (MTT) calculando el porcentaje de citotoxicidad (\%CC).

$\% \mathrm{CC}=\frac{\text { Densidad óptica (DO) grupo control }- \text { DO grupo tratado }}{\text { DO grupo control }} \times 100$

La actividad de los compuestos fue expresada como la concentración inhibitoria o citotóxica para el $50 \%$ de las células $\left(\mathrm{Cl}_{50}\right.$ y $\left.\mathrm{CC}_{50}\right)$ calculada por análisis de regresión sigmoidal ${ }^{(13)}$.

\section{ENSAYOS IN VIVO}

Los ratones fueron infectados con promastigotes en fase estacionaria ( $5 \times 10^{5}$ parásitos) en el dorso. Después de 8-12 semanas (lesiones aproximadamente de $30 \mathrm{~mm}^{2}$ ), los animales fueron tratados con una dosis diaria de cada SF$\mathrm{KTZ}$, sobre las lesiones por 60 días.

Se conformaron 13 grupos con dos ratones en cada uno. Todos los tratamientos fueron evaluados en ratones infectados con $L$. (V.) braziliensis. Adicionalmente en los grupos del 8 al 13 se incluyeron ratones infectados con $L$. (V.) panamensis. El total se utilizaron 38 ratones, de acuerdo con las siguiente secuencia y tratamiento:

Grupo 1-5: diez ratones tratados con SF-KTZ tipo gel (gel 1-gel 4 y gel sin KTZ).

Grupo 6-7: cuatro ratones tratados con lipogel, con y sin KTZ. Grupo 8-12: 20 ratones tratados con SF-KTZ tipo crema (diferentes porcentajes de KTZ y crema sin KTZ).

Grupo 13: cuatro ratones tratados con miltefosina.

Semanalmente se midieron las lesiones (nódulo y úlcera) utilizando un medidor digital. Los ratones se sacrificaron 15 días después de terminar el tratamiento utilizando una mezcla de anestésicos (Ketamina/Xilacina) vía intraperitoneal. Se realizaron extendidos en láminas portaobjetos de las lesiones y se recolectaron biopsias de las lesiones, hígado y bazo. Se comparó el tamaño de las lesiones $\left(\mathrm{mm}^{2}\right)$ con el vehículo y la presencia/ausencia de parásitos en los extendidos y biopsias. Los fragmentos de órganos fueron fijados con formalina al $10 \%$ por 24 horas y posteriormente incluidos en bloques de parafina. Se realizaron cortes de cinco $\mu \mathrm{m}$ los cuales fueron coloreados con hematoxilina-eosina.

\section{PRUEBA DE IRRITACIÓN}

Fue realizada según el método descrito en la prueba número 404 Irritación/Corrosión Dérmica Aguda de la Organización para la Cooperación y el Desarrollo Económico (OECD) (https://goo.gl/L28Duh). Los ratones sanos fueron tratados tópicamente con cada SF-KTZ por siete días. Las lesiones se clasificaron como 0 (sin eritema), 1 (eritema ligero), 2 (eritema bien definido), 3 (eritema moderado o severo) y 4 (eritema grave).

\section{ANÁLISIS ESTADÍSTICO}

Se utilizó la prueba de ANOVA de una vía para comparar los diferentes grupos. Un valor de $p<0,05$ fue considerado estadísticamente significativo.

\section{CONSIDERACIONES ÉTICAS Y MANTENIMIENTO DE ANIMALES}

El presente trabajo contó con el aval del Comité de Ética de la Universidad Industrial de Santander, código 2016-P10CI015. Los ratones fueron mantenidos a $20 \pm 3^{\circ} \mathrm{C}$, en fotoperiodos de luz/oscuridad de 12 horas, con alimento y agua ad libitum.

\section{HALLAZGOS}

\section{CARACTERÍSTICAS FISICOQUIMMICAS DE LOS SF-KTZ}

Los SF-KTZ tipo gel fueron de color blanco, aspecto homogéneo, $\mathrm{pH}$ 5-6 y extensibilidad del 0,78 a 1,28 siendo mayor en el Gel 4 (1,5\%) (gel sin PPP). Las cremas de KTZ mostraron un color blanco, aspecto homogéneo, $\mathrm{pH} 7$ y extensibilidad entre 0,5 y 0,78 siendo la menos extensible la crema $3(15 \%)$.

Los geles, lipogeles y cremas fueron estables por 30 días a $4{ }^{\circ} \mathrm{C}$ y $28^{\circ} \mathrm{C}$ en oscuridad. El gel 1 y 2 y las cremas cambiaron de color a anaranjado-rosado a $37^{\circ} \mathrm{C}$.

El tamaño, potencial $Z$ y PDI en los LUD de KTZ fue de $86,26 \pm 1,32 \mathrm{~nm},-18,6 \pm 0,85 \mathrm{mV}$ y 0,135 , respectivamente, y en los LUD vacíos fue de $119,3 \pm 2,95 \mathrm{~nm},-35,3 \pm 1,92$ $\mathrm{mV}$ y 0,156 , respectivamente. Fueron estables a $4^{\circ} \mathrm{C}$ hasta por 30 días.

\section{ACTIVIDAD IN VITRO}

Los promastigotes fueron susceptibles al tratamiento con los SF-KTZ tipo crema conteniendo KTZ al $2 \%, 10 \%$ y $15 \%$, con actividades de $\mathrm{Cl}_{50}$ de $<11,10 \mu \mathrm{g} / \mathrm{mL}, 5,81 \mu \mathrm{g} / \mathrm{mL}$ y $7,61 \mu \mathrm{g} / \mathrm{mL}$, respectivamente. Ninguno de los SF-KTZ (a nivel del $\mathrm{Cl}_{50}$ ) mostró actividad en amastigotes intracelulares. La toxicidad en células THP-1 de las cremas en general fue baja con valores de $\mathrm{CC}_{50}$ de $403,05 \mu \mathrm{g} / \mathrm{mL}, 121,06 \mu \mathrm{g} / \mathrm{mL}$ y $121,73 \mu \mathrm{g} / \mathrm{mL}$, a concentraciones de KTZ de $2 \%, 10 \%$ y 
$15 \%$, respectivamente. EI KTZ libre (en solución) mostró actividad en promastigotes y amastigotes intracelulares $\left(\mathrm{Cl}_{50}\right.$ $<5,03 \mu \mathrm{g} / \mathrm{mL}$ y $6,12 \mu \mathrm{g} / \mathrm{mL}$, respectivamente) y células $\left(\mathrm{CC}_{50}\right.$ $32,5 \mu \mathrm{g} / \mathrm{mL}$ ) (Figura 1). Los vehículos no mostraron actividad contra parásitos ni células THP-1 (datos no incluidos).

\section{EFECTIVIDAD DE LAS FORMULACIONES DE KTZ EN RATONES}

Ninguno de los tratamientos fue efectivo en ratones con LC (Figura 2 y 3). Después del tratamiento, las lesiones de los ratones infectados con $L$. (V.) braziliensis se incrementaron de $23,1 \%$ a $96,7 \%$ y en los ratones infectados con $L$. (V.) panamensis se incrementaron de $45,17 \%$ a $89,26 \%$, similar al incremento en los controles. La evolución de las lesiones al tratamiento con los SF-KTZ tipo crema se muestran en la Figura 2. Se observaron parásitos en todos los extendidos y biopsias de lesiones. No se encontraron parásitos en las biopsias de bazo e hígado. No se observaron cambios en el peso corporal o en la ingesta de alimentos y agua. El tratamiento tópico con miltefosina fue efectivo en los ratones tratados.

\section{IRRITACIÓN DE LOS TRATAMIENTOS}

Ninguno de los tratamientos fue irritante para los ratones.

\section{DISCUSIÓN}

El tratamiento tópico con KTZ en LC no ha mostrado resultados satisfactorios ${ }^{(8-10)}$. Algunas de sus características fisicoquímicas podrían afectar su absorción y difusión hacia las capas profundas de la piel y por ende su efectividad. EI KTZ es una base débil con baja solubilidad en agua y alto peso molecular. Se sabe que compuestos de bajo peso molecular presentan una mejor difusión a través del estrato córneo de la piel ${ }^{(14)}$. Sin embargo, su baja solubilidad en agua y relativa efectividad a bajas concentraciones in vitro $\left(\mathrm{Cl}_{50} 6,12 \mu \mathrm{g} / \mathrm{mL}\right)$ podrían constituir factores a su favor, por lo que este fármaco incluido en SF adecuados podría mostrar una mejor absorción y difusión hacia las capas profundas de la piel ${ }^{(14)}$.

A diferencia del tratamiento tópico con miltefosina utilizado como control, los SF utilizados no fueron efectivos en LC. Sin embargo, se lograron formulaciones estables, de características fisicoquímicas recomendadas para un tratamiento tópico, capaces de incluir diversos porcentajes del fármaco y PPP reconocidos (dimetilsulfoxido, ácido oleico o un terpeno) y de mantener la actividad del fármaco in vitro contra los parásitos.

La presencia del dimetilsulfoxido (DMSO) era una opción importante. Este es un compuesto no tóxico y seguro, con

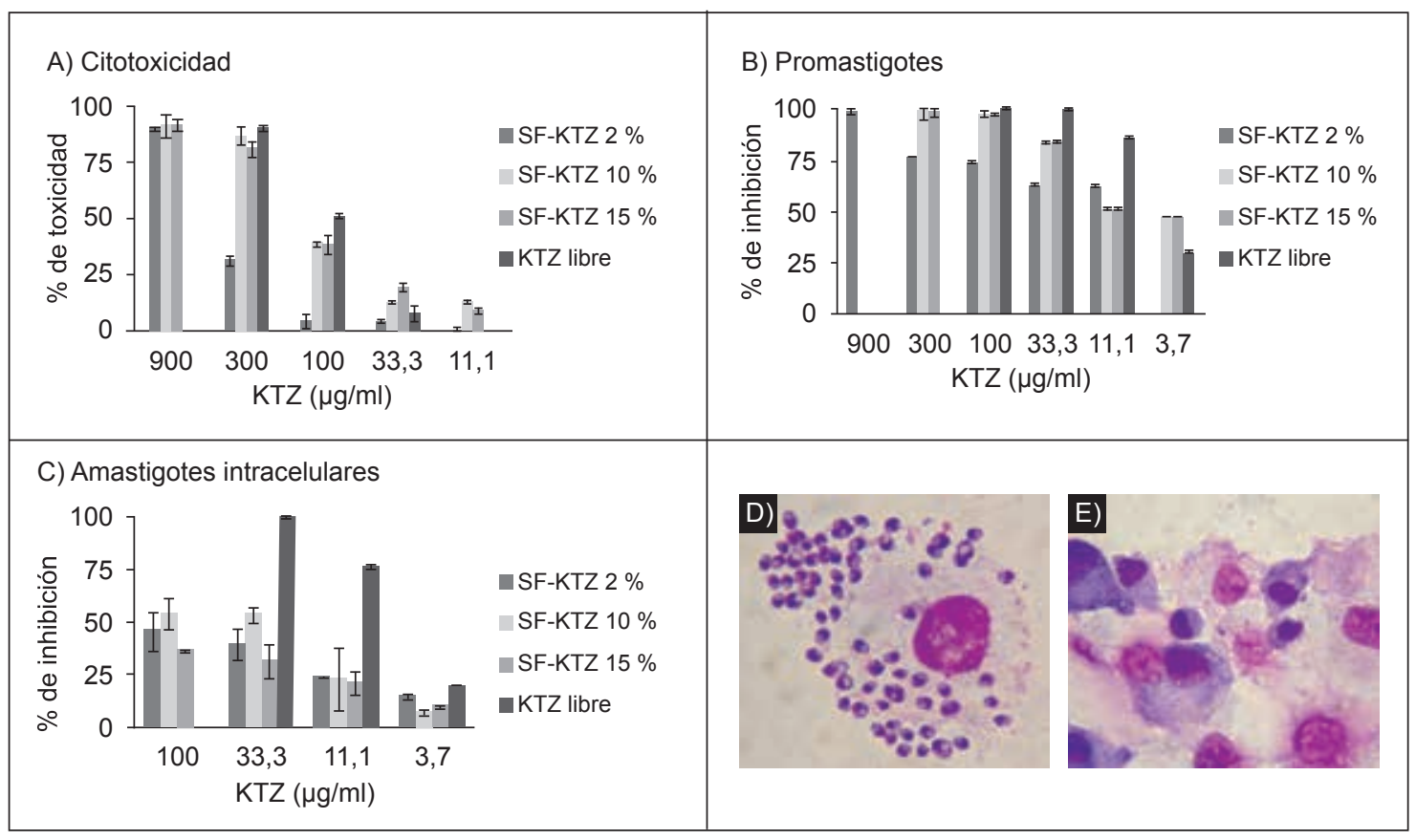

KTZ: ketoconazol, SF-KTZ: Sistema farmacéutico de ketoconazol, KTZ libre: ketoconazol sin la crema, en solución

Figura 1. Actividad in vitro de los sistemas farmacéuticos de ketoconazol tipo crema. (A) Citotoxicidad de los sistemas y del ketoconazol libre (en solución) en monocitos transformados a su fenotipo adherente THP-1 (B) en promastigotes y (C) en amastigotes intracelulares de L. (V.) brazilinesis. (D) Microfotografía de una célula THP-1 infectada con L. (V.) braziliensis coloreada con Giemsa, aumento 1000X (E) Microfotografía de las células THP-1 después del tratamiento con ketoconazol, coloreadas con Giemsa, aumento 1000X 


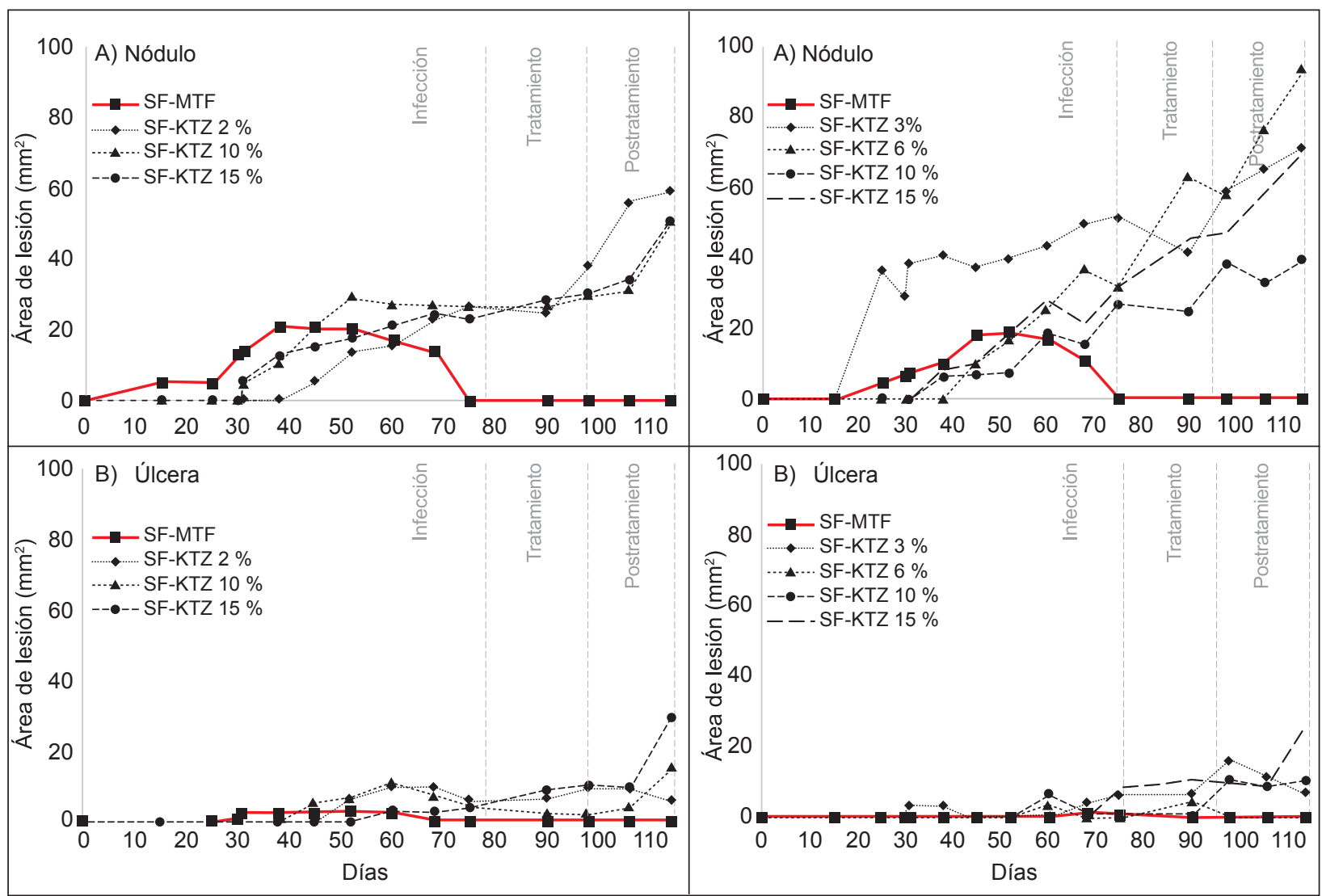

SF-MTF: Sistema farmacéutico de miltefosina, SF-KTZ: Sistema farmacéutico de ketoconazol

Figura 2. Eficacia del tratamiento con los sistemas farmacéuticos de ketoconazol tipo crema en ratones BALB C infectados con: $L$. (V.) braziliensis y L. (V.) panamensis. Evolución de A) nódulo y B) úlcera durante la infección, tratamiento y postratamiento. Los ratones controles fueron tratados con un gel de miltefosina por 20 días (línea roja). El tratamiento con los SF-KTZ fue realizado por 60 días

actividad a nivel de los fosfolípidos de la bicapa lipídica, utilizado en algunos SF tópicos; por ejemplo, en diclofenaco sódico o en LC de viejo mundo en donde una formulación de KTZ al $2 \%$ y DMSO al $20 \%$, aplicado cinco veces al día por un mes mostró una eficacia del $89 \%$, induciendo una destrucción más rápida de los parásitos en las lesiones ${ }^{(15)}$.

Se obtuvieron vesículas (LUD tipo transferosoma) homogéneas, nanométricas y estables las que fueron incluidas uniformemente en un gel; sin embargo, tampoco fueron eficaces. Este tipo de liposomas (LUD-miltefosina) fueron preparados previamente por nuestro grupo induciendo una mayor liberación del fármaco en piel en comparación con el preparado no encapsulado ${ }^{(13)}$. Otros sistemas vesiculares, microemulsiones y nanopartículas conteniendo KTZ han sido preparados mejorando su eficacia antifúngica y permitiendo una mayor permeación del fármaco en piel ${ }^{(15-20)}$. Estos sistemas coloidales podrían ser candidatos para ser evaluados en LC.

Dada la ineficacia de las cremas de KTZ en los ratones infectados con L. (V.) braziliensis, se decidió evaluarlas en
L. (V.) panamensis, esto debido a la especificidad de la especie, mostrada en ensayos clínicos con KTZ oral (6-7). Los resultados fueron igualmente negativos.

Es importante mencionar, como limitación, que sólo se incluyeron dos ratones por grupo; esto debido a la dificultad del modelo utilizado en la generación de lesiones (sólo en el $40 \%$ de los infectados), sin embargo, dada las características de las lesiones (nodulares grandes, ulceradas, con alta concentración de parásitos, incapaces de curar espontáneamente), en las que se utilizaron SF-KTZ con diferentes concentraciones de fármaco (1-15\%), pensamos que los resultados obtenidos nos brindan indicios de la poca eficacia del KTZ en el tratamiento de la LC producida por estas dos especies de Leishmania.

En conclusión, los SF-KTZ administrados tópicamente no fueron eficaces frente a la LC causada por $L$. (V.) braziliensis y $L$. (V.) panamensis en los ratones utilizados. Estudios de permeación del KTZ in vivo utilizando otros sistemas farmacéuticos o PPP que permitan una mejor absorción del $\mathrm{KTZ}$ en piel son recomendados. 
A) L. (V.) braziliensis

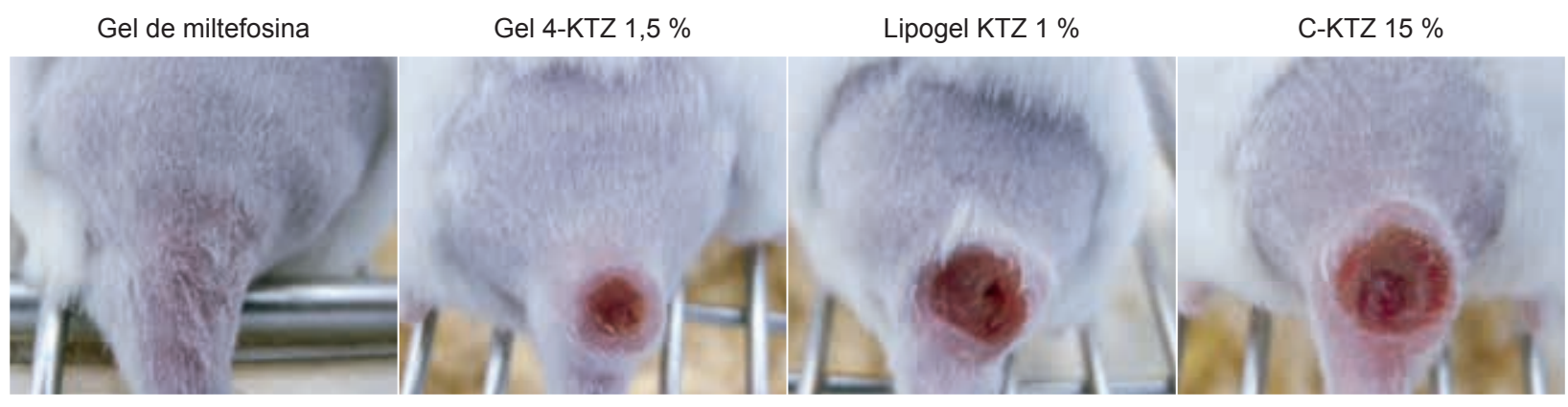

B) L. (V.) panamensis

Gel de miltefosina

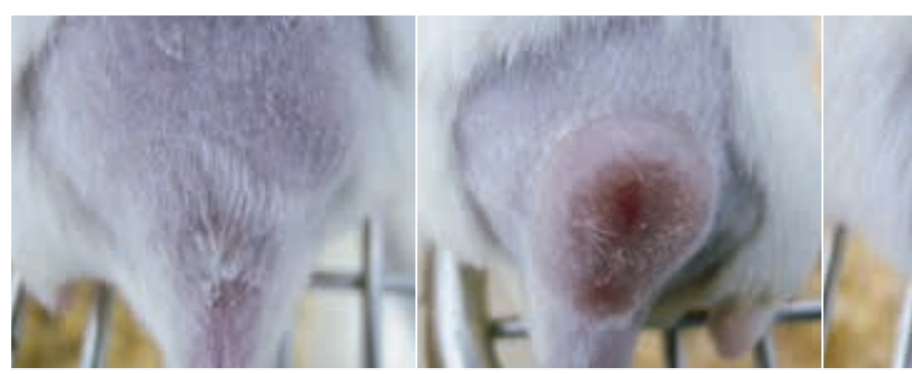

C-KTZ $10 \%$

C-KTZ $15 \%$

KTZ: ketoconazol; lipogel: liposomas + gel; C: crema.

Figura 3. Efecto los tratamientos tópicos en las lesiones de leishmaniasis cutánea. Las fotografías muestran las lesiones resultantes después de 15 días de tratamiento con los diferentes sistemas farmacéuticos de ketoconazol en ratones BALB C infectados con: A) $L$. (V.) braziliensis y B) $L$. (V.) panamensis. En la primera foto de cada fila, se muestra los resultados del tratamiento con miltefosina tópica

Agradecimientos: Agradecemos a la Vicerrectoría de Investigación y Extensión de la Universidad Industrial de Santander y a COLCIENCIAS por el apoyo en la realización del presente proyecto.

Contribuciones de los autores: AMV y PE participaron en el desarrollo y diseño de los experimentos. OAC participó en los experimentos realizados in vivo en $L$. (V.) panamensis. JCM participó en el análisis e interpretación de los estudios histopatológicos. Todos participaron en la redacción del artículo y aprobaron la versión final.
Fuentes de financiamiento: La presente investigación fue financiada con recursos de la Vicerrectoría de Investigación y Extensión de la Universidad Industrial de Santander UIS, proyecto Código 1850 y con recursos del Patrimonio Autónomo Fondo Nacional de Financiamiento para la Ciencia la Tecnología y la Innovación Francisco José de Caldas, Contrato RC-687-2014.

Conflictos de interés: Los autores declaran no tener conflicto de intereses en la realización de este trabajo.

\section{REFERENCIAS BIBLIOGRÁFICAS}

1. AlvarJ,VélezI,Bern C,HerreroM,Desjeux $\mathrm{P}$, Cano J et al. Leishmaniasis worldwide and global estimates of its incidence. PLoS ONE. 2012; 7(5):e35671. doi: 10.1371/ journal.pone.0035671.

2. Rodríguez G, Arenas C, Ovalle C, Hernández C. Las Leishmaniasis: atlas y texto. 1era edición. Bogotá: Editorial Panamericana; 2016 p. 91-106.

3. Blum J, Lockwood D, Visser L, Harms G, Bailey M, Caumes E et al. Local or systemic treatment for New World cutaneous leishmaniasis? Re-evaluating the evidence for the risk of mucosal leishmaniasis. Int Health. 2012; 4(3):15363. doi: 10.1016/j.inhe.2012.06.004.
4. Food and Drug Administration [Internet]. Maryland: FDA; 2017. [Citado el 22 de febrero 2018]. FDA Drug Safety Communication: FDA limits usage of Nizoral (ketoconazole) oral tablets due to potentially fatal liver injury and risk of drug interactions and adrenal gland problems; [dos pantallas] Disponible en https://www.fda. gov/Drugs/DrugSafety/ucm362415.htm

5. McCall LI, El Aroussi A, Choi JY, Vieira DF, De Muylder G, Johnston JB, et al. Targeting ergosterol biosynthesis in Leishmania donovani: essentiality of sterol 14 alpha-demethylase PLoS Negl Trop Dis. 2015;9(3):e0003588. doi: 10.1371/ journal.pntd.0003588.
6. Saenz RE, Paz H, Berman JD. Efficacy of ketoconazole against Leishmania braziliensis panamensis cutaneous leishmaniasis. Am J Med. 1990; 89(2):147-55.

7. Navin TR, Arana BA, Arana FE, Berman JD, Chajón JF. Placebo-controlled clinical trial of sodium stibogluconate (Pentostam) versus ketoconazole for treating cutaneous leishmaniasis in Guatemala. J Infect Dis. 1992; 165(3):528-34.

8. Restrepo M, Gomez M. Tratamiento de la leishmaniasis cutanea con ketoconazol. Acta Médica Colomb. 1987;12(4):294-7.

9. Abahusein A, Larbi E, al-Khawajah A, al-Gindan Y, Jain S. Evaluation of topical 
ketoconazole in cutaneous leishmaniasis. East Afr Med. 1992; 69(1):14-7.

10. Momeni AZ, Aminjavaheri M, Omidghaemi MR. Treatment of cutaneous leishmaniasis with ketoconazole cream. J Dermatolog Treat. 2003;14(1):26-9.

11. Scheinfeld N. Ketoconazole: a review of a workhorse antifungal molecule with a focus on new foam and gel formulations. Drugs Today (Barc). 2008; 44(5):369-80. doi: 10.1358/dot.2008.44.5.1216598

12. Kaur IP, Kakkar S. Topical delivery of antifungal agents. Expert Opin Drug Deliv. 2010; 7(11):1303-27. doi: 10.1517/17425247.2010.525230.

13. Hernandez IP, Montanari JAM, Escobar P. Actividad in vitro contra Leishmania y permeación en piel humana de liposomas ultradeformables de miltefosina. Rev Cuba Med Trop 2014; 66 (3):370-85.

14. Durand C, Alhammad A, Willett KC. Practical considerations for optimal transdermal drug delivery. Am J Health Syst
Pharm. 2012; 15;69(2):116-24. doi 10.2146/ajhp110158.

15. Dutz W, Agarval N, Bashardost MZ, Bentley G, Kindmark CO. Topical therapy of cutaneous leishmaniasis with 2\% ketoconazole ointment and DMSO. Int J Dermatol. 1987; 26(3):199.

16. Guo F, Wang J, Ma M, Tan F, Li N. Skin targeted lipid vesicles as novel nano-carrier of ketoconazole: characterization, in vitro and in vivo evaluation. J Mater Sci Mater Med. 2015; 26(4):175. doi: 10.1007/ s10856-015-5487-2.

17. Shirsand S, Para M, Nagendrakumar D, Kanani K, Keerthy D. Formulation and evaluation of Ketoconazole niosomal gel drug delivery system. Int J Pharm Investig. 2012; 2(4):201-7. doi: 10.4103/2230973X.107002.

18. Che J, Wu Z, Shao W, Guo P, Lin Y, Pan $\mathrm{W}$, et al. Synergetic skin targeting effect of hydroxypropyl- $\beta$-cyclodextrin combined with microemulsion for ketoconazole. Eur
J Pharm Biopharm. 2015; 93:136-48. doi: 10.1016/j.ejpb.2015.03.028.

19. Ramasamy T, Khandasami U, Ruttala $\mathrm{H}$, Shanmugam S. Development of solid lipid nanoparticles enriched hydrogels for topical delivery of anti-fungal agent. Macromol Res. 2012; 20(7):682-92.

20. Patel MR, Patel RB, Parikh JR, Solanki $A B$, Patel BG. Investigating effect of microemulsion components: In vitro permeation of ketoconazole. Pharm Dev Technol. 2011;16(3):250-8. doi: $10.3109 / 10837451003610845$.

\section{Correspondencia: Patricia Escobar}

Dirección: Km 2 Via el Refugio, Sede UIS Guatiguará. Bucaramanga, Colombia, Teléfono: (57) (7) 634400 Ext 3528,

Correo electrónico:pescobarwww@yahoo.co.uk

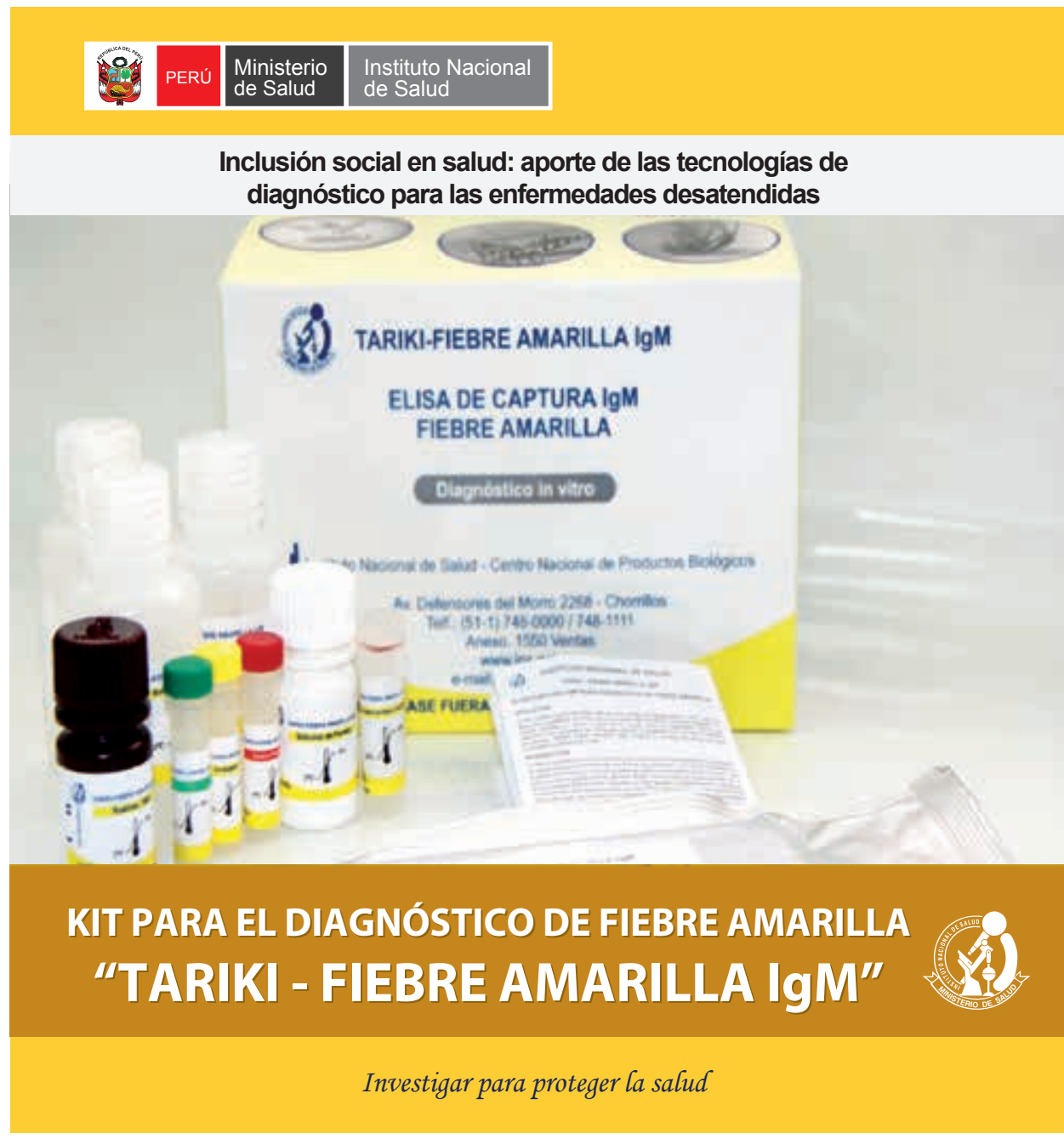

